\title{
THE SUMMIT OF THE CBR AT MM WAVELENGTHS AND OTHER QUERIES
}

\author{
J. P. Vallee \\ Herzberg Institute, NRCC, Ottawa, Ont., Canada K1A OR6
}

\section{$\underline{\text { Summary }}$}

As a strategy for the Search for Extraterrestrial Intelligence (SETI), a renewed proposal is made to look at the summit of the Cosmic Background Radiation (CBR). The CBR peaks near $1.85 \mathrm{~mm}$ in the frequency spectrum of the Universe and near $1.05 \mathrm{~mm}$ in the wavelength spectrum of the Universe. I review the current status (best CBR temperature, best receivers, etc).

In addition, a modelling approach to existentialist questions and SETI is developed briefly, as a game. Existentialist "answers" (religious, scientific) are tabulated for questions on the Universe, Life, the Mind, according to their various choices. 